\title{
Magnetized Viscous Fluid Anisotropic Models in String Cosmology
}

\author{
C.P.Singh ${ }^{1, *}$, A. Beesham ${ }^{2}$ \\ ${ }^{1}$ Department of Applied Mathematics, Delhi Technological University (Formerly Delhi College of Engineering), \\ Bawana Road, Delhi-110 042, India \\ ${ }^{2}$ Department of Mathematical Sciences, University of Zululand, Private Box. X1001, Kwa-Dlangezwa 3886, South Africa \\ *Corresponding Author: cpsphd@rediffmail.com
}

Copyright (C2014 Horizon Research Publishing All rights reserved.

\begin{abstract}
We study the evolution of a homogeneous and anisotropic Bianchi type $\mathrm{VI}_{0}$ cosmological model in the presence of magnetic field and viscous fluid together with a cloud of cosmic strings in general relativity. Since Viscous fluid and magnetic field have cosmological origin, it is interesting to discuss the effects of viscous fluid and magnetic field on the expansion history of the universe in early and later stages of evolution in string cosmology. Exact solutions of the field equations are obtained using the equation of state for a cloud of strings. The bulk viscous coefficient is assumed to be inversely proportional to the expansion scalar. The two string models, namely, geometrical(Nambu string) and Takabayasi (p-string) string models are discussed with magnetized and non-magnetized viscous fluid by taking certain physical conditions. We also examine numerically the effects of bulk viscosity and magnetic field on potential in each string model. We find that magnetic field and viscous fluid play important role during the early evolution of the universe. We observe that the viscous term affects the evolution much rapid as compare to magnetic field. The presence of viscous term prevents the universe to be empty. The other physical and geometrical aspects of each string model are also discussed in detail.
\end{abstract}

Keywords cosmology; Magnetic field; Bulk Viscosity

PACS number(s): 98.80-k, 98.80-cq, 04.20-q

\section{Introduction}

In recent years cosmic strings have been studied to describe the early evolution of the universe. Kibble ${ }^{1}$, Zeldovich ${ }^{2}$ and Vilenkin ${ }^{3}$ believed that cosmic strings might be the source of density perturbations which are required for the formation of large scale structures in the universe. In the early stages of evolution of the universe it is expected that topological defects could have formed naturally during the phase transitions followed by spontaneous broken symmetries. Cosmic strings are linear topological defects, have very interesting properties which play an important role in the structure formation. These cosmic strings have stress energy and couple to the gravitational field. The gravitational effect of string in general relativity have been studied by Letelier ${ }^{4}$ and Stachel ${ }^{5}$. Letelier ${ }^{6}$ studied relativistic cosmological solutions of cloud formed by massive strings in Bianchi type-I and Kantowski-Sachs space-times. In these models each massive string is formed by a geometric string with particles attached along its extension. Letelier ${ }^{4}$ pointed out that the universe could be represented by a collection of objects (galaxies), so a string dust cosmology gives a model to investigate properties related with this fact. Zeldovich ${ }^{2}$ also explained that the string dust model could be used as a source of the gravitational field. In geometrical string model, the strings dominate and the tension along the string is equal to its energy density per unit length as we call it string dust model. Since the presence of strings in the early universe can be explained using grand unified theories, there must be different kinds of vacuum structures depending on the structure and topology of the gauge group. Letelier ${ }^{6}$ studied a model of a cloud formed by massive strings instead of geometrical strings. Each massive string is formed by a geometrical strings with particles attached along its extension. Hence, the strings that form the cloud are the generalization of Takabayasi's realistic model of strings (p-strings). Therefore, p-string is the direct consequence of Takabayasi's string model. In principle we can eliminate the strings and end up with a cloud of particles. This is a desirable property of a model of a string cloud to be used in cosmology. The different string models can be represented by an equation of state of a cloud of strings. Matraverse ${ }^{7}$ presented a class of exact solutions of 
Einstein field equations with a two parameter family of classical strings as the source of the gravitational field.

The role of viscosity as a dissipative mechanism may be important in cosmology for a number of reasons. The dissipative mechanism not only modifies the nature of singularity usually occurring for perfect fluid but also successfully accounts for the large entropy per baryon in the present universe. Misner ${ }^{8,9}$, while discussing the electron -neutrinos scattering and the subsequent decoupling of neutrinos in Bianchi type -I models, came to the conclusion that the viscosity of neutrinos could essentially reduce the initial anisotropy of the universe. Matzner ${ }^{10}$ came to the similar conclusion for the Bianchi type -V models. A critical analysis to Misner's method were raised by Doroskevich et al. ${ }^{11}$, Stewart ${ }^{12}$, and Collins and Stewart ${ }^{13}$. They found that the isotropization by decoupling neutrinos could have lead to the isotropy level now observed only if at the end of the lepton era the degree of anisotropy was already small.

It is interesting to note that magnetic field present in galactic and inter galactic spaces play a significant role at cosmological scale. The study of magnetic field in the matter distribution is of considerable interest as it provides an effective way to understand the initial phases of cosmic evolution. The inclusion of the magnetic field is motivated by the observational cosmology and astrophysics indicating that many subsystems of the universe possess magnetic fields. It is reasonable to consider magnetic fields in the energy momentum tensor of the early universe. A cosmological model containing a global magnetic field is necessarily anisotropic. An understanding of the effect of a magnetic field upon the dynamics of the universe is necessary during early and late time evolution of the universe. Melvin ${ }^{14}$ suggested that during the evolution of the universe, the matter was in a highly ionized state and was smoothly coupled to the gravitational field and subsequently forming neutral matter during expansion of the universe. Primordial magnetic field of cosmological origin have been discussed by Asseo and Sol ${ }^{15}$, and Madsen ${ }^{16}$. Wolfe et al. ${ }^{17}$, Kulsrud et al. ${ }^{18}$, Barrow ${ }^{19}$, Matravers and Tsagas ${ }^{20}$ are some of the authors who have studied the cosmological models with magnetic field and have pointed out its important in the early evolution of the universe. Therefore, in string dust universe the presence of magnetic field is not unrealistic.

The recent observational data from Cosmic Background Explorer (COBE) and Wilkinson Microwave Anisotropy Probe (WMAP) support the existence of an anisotropic phase in the evolution of the universe. These studies lead many cosmologists to consider the anisotropic models to describe the early phase of the evolution of the universe which decay into isotropic in late time. Homogeneous and anisotropic cosmological models have been widely studied in the framework of general relativity to describe the early and late phases of the evolution of the universe. These models have a significant role in the description of the universe at early stages of the evolution. Therefore, it is interesting to study anisotropic cosmological models that decay into isotropic in later stages of evolution of the universe. Bianchi type $-\mathrm{VI}_{0}\left(\mathrm{BVI}_{0}\right)$ space times are of particular interest since they are sufficiently complex, while at the same time, they are simple generalizations of Bianchi type spaces. Barrow ${ }^{21}$ pointed out that $\mathrm{BVI}_{0}$ cosmological model give a better explanation of some of the cosmological problems like primordial helium abundance and isotropization in a special sense. Some of the authors ${ }^{22-32}$ have discussed $\mathrm{BVI}_{0}$ cosmological models with matter distribution in general relativity and scalar tensor theory.

Weaver ${ }^{33}$ has studied dynamics of magnetic $\mathrm{BVI}_{0}$ cosmologies whereas Sharif and Zubair ${ }^{34,35}$ have discussed the dynamics of $\mathrm{BVI}_{0}$ with and without magnetic field in the presence of anisotropic fluid. In recent past, many authors ${ }^{36-38}$ have studied cosmic strings in Bianchi type $\mathrm{VI}_{0}$ space time in general relativity. Ribeiro and Sanyal ${ }^{39}$, Banerjee and Sanyal ${ }^{40}$, Bali and Jain ${ }^{41}$ have studied $\mathrm{BVI}_{0}$ model with bulk viscosity and magnetic field in general relativity. Wang ${ }^{42,43}$, Yadav et al. ${ }^{44}$, Bali et al. ${ }^{45,46}$, Tripathy et al. ${ }^{47,48}$, Tripathy and Behera ${ }^{49}$ have studied $\mathrm{BVI}_{0}$ string cosmological models with magnetic field and viscous fluid in general relativity. Pradhan and Bali ${ }^{50}$ have studied $\mathrm{BVI}_{0}$ massive string cosmological models with decaying vacuum energy density. Sharif and Waheed ${ }^{51}$ have discussed the dynamics of magnetized bulk viscous strings in Brans-Dicke gravity. Saha and Visinescu ${ }^{52}$ have investigated the effect of magnetic field on classical potential in string cosmology. Saha et al. ${ }^{53}$ and Rikhvitsky et al. ${ }^{54}$ have studied Bianchi I string cosmological models in the presence of magnetic field and have examined the quantum effects in loop quantum cosmology. Saha and Visinescu ${ }^{55}$ have extended their work to study the effect of magnetic field in string cosmology for Bianchi VI cosmological model. Recently Singh ${ }^{56,57}$ has studied Bianchi I with viscous fluid and Bianchi V string cosmological models in the presence of magnetic field.

The presently observed universe is almost isotropic at large as we have discussed above, therefore, the isotropization problem appears inevitably in any study of anisotropic cosmologies. The presence of such a field demands the use of a class of space -times more general than the Friedmann - Roberstion-Walker ones, since the isotropy is broken. The most natural extension is the study of homogeneous and anisotropic models. String cosmological model have been used in attempts to describe the early universe and to investigate anisotropic behavior. On the other hand, bulk viscosity lead to negative energy field and hence have a significant impact on the dynamics of the universe. The motivation of this paper comes from the above discussion. It would be worthwhile to discuss the early and late phases of the universe in $\mathrm{BVI}_{0}$ magnetized bulk viscous string cosmological model in general relativity. The main purpose of this work is to examine the effects of bulk viscosity on the dynamics of the universe with and without magnetic field in $\mathrm{BVI}_{0}$. Assuming certain assumptions, some exact solutions of the field equations are presented for different string models. It is also resealable to study the string model in late time evolution.

The paper is organized as follows: In Section 2 we present spatially homogeneous and anisotropic $\mathrm{BVI}_{0}$ space time and derive the field equations for cosmic string with magnetic field and viscous fluid. Section 3 deals with the exact solutions for different string models and discuss the physical behavior of bulk viscosity on the evolution of the universe with and without magnetic field. Finally, Section 4 concludes the results. 


\section{Model and Field Equations}

The spatially homogeneous and anisotropic Bianchi type $\mathrm{VI}_{0}$ model is described by the line element

$$
d s^{2}=d t^{2}-A^{2} d x^{2}-e^{2 m x} B^{2} d y^{2}-e^{-2 m x} C^{2} d z^{2},
$$

where $A, B$, and $C$ are functions of cosmic time $t$ only and are the scale factors in anisotropic background, and $m \neq 0$ is a constant.

It is useful to define the volume scale factor $\tau$

$$
\tau=A B C .
$$

The mean expansion rate as an average Hubble rate $H$ is given by

$$
H=\frac{1}{3}\left(\frac{\dot{A}}{A}+\frac{\dot{B}}{B}+\frac{\dot{C}}{C}\right)=\frac{1}{3} \frac{\dot{\tau}}{\tau}
$$

where an overdot means derivative with respect to time $t$. The expansion scalar $\theta$ is defined by

$$
\theta=u_{; l}^{l}=\left(\frac{\dot{A}}{A}+\frac{\dot{B}}{B}+\frac{\dot{C}}{C}\right)=\frac{\dot{\tau}}{\tau} .
$$

We also define the two relative shear anisotropy parameters $R$ and $S$, i.e., the differences of the expansion rates as the Hubble normalized shear ${ }^{58}$ as

$$
R=\frac{1}{H}\left(\frac{\dot{A}}{A}-\frac{\dot{B}}{B}\right), \quad S=\frac{1}{H}\left(\frac{\dot{A}}{A}-\frac{\dot{C}}{C}\right) .
$$

When $R=0=S$ and $m=0$, the line element (1) reduces to Friedmann -Robertson - Walker (FRW) model. The energy momentum tensor of a cloud of strings in presence of bulk viscosity and magnetic field is given as ${ }^{18,59,60}$

$$
T_{i}^{j}=\rho u_{i} u^{j}-\lambda x_{i} x^{j}-\xi u^{l} ;_{l}\left(g_{i}^{j}+u_{i} u^{j}\right)+E_{i}^{j},
$$

where $\rho$ is the rest energy density for a cloud of strings with particles attached to them and $\lambda$ is the string tensor density which may be negative, zero or positive and is related by $\rho=\rho_{p}+\lambda$, where $\rho_{p}$ is the particle energy density. The unit time-like vector $u^{i}$ describes the particle's four velocity and unit space-like vector $x^{i}$ denotes the direction of the string which can be taken along any of the three directions $x, y$ and $z$ axes. Thus, without loss of generality let us choose $x$ - direction as the direction of the string along which the magnetic field is assumed to be present, i.e.,

$$
x^{i}=\left(A^{-1}, 0,0,0\right)
$$

In a co-moving coordinate system, we have $u^{i}=(0,0,0,1)$. Therefore, $u_{i}$ and $x_{i}$ satisfy the conditions

$$
u_{i} u^{i}=-x_{i} x^{i}=-1, \quad u_{i} x^{i}=0 .
$$

In Eq. (6), $\xi$ is the coefficient of bulk viscosity and $E_{i}^{j}$ is the electromagnetic field tensor which is given by (see, ref. $\left.^{60}\right)$

$$
E_{i}^{j}=\bar{\mu}\left[|h|^{2}\left(u_{i} u^{j}+\frac{1}{2} g_{i}^{j}\right)-h_{i} h^{j}\right],
$$

where $\bar{\mu}$ is the magnetic permeability, and $h_{i}$, the magnetic flux vector defined by

$$
h_{i}=\frac{\sqrt{-g}}{2 \bar{\mu}} \epsilon_{i j k l} F^{k l} u^{j} .
$$

Here $F^{k l}$ is the electromagnetic field tensor and $\epsilon_{i j k l}$ is the Levi-Civita tensor density.

We assume that there is a magnetic field along $x$ - direction, that is, along the direction of string. The Maxwell's equations

lead to

$$
F_{i j ; k}+F_{j k ; i}+F_{k i ; j}=0, \text { and } F_{; k}^{i j}=0,
$$

$$
F_{23}=I \text {, }
$$

where $I$ is a constant characterizing the magnetic field intensity. It is to be noted that the semicolon stands for covariant derivative. All other components of $F^{k l}$ are zero.

It follows from Eq.(10) that the non-zero component of magnetic flux vector is

$$
h_{1}=\frac{I A}{\bar{\mu} B C} .
$$


Using (13) into (9), the non-trivial components of $E_{i}^{j}$ are given by

$$
E_{1}^{1}=-\frac{I^{2}}{2 \bar{\mu}^{2} B^{2} C^{2}}=-E_{2}^{2}=-E_{3}^{3}=E_{4}^{4} .
$$

The Einstein's field equations (in gravitational units $c=8 \pi G=1$ ) read as

$$
R_{i}^{j}-\frac{1}{2} g_{i}^{j} R=-T_{i}^{j}
$$

where $R_{i}^{j}$ is the Ricci tensor and $R=g^{i j} R_{i j}$ is the Ricci scalar.

The field equations (15) with (1) and (6) lead to the following system of equations

$$
\begin{gathered}
\frac{\dot{A} \dot{B}}{A B}+\frac{\dot{B} \dot{C}}{B C}+\frac{\dot{C} \dot{A}}{C A}-\frac{m^{2}}{A^{2}}=\rho+\frac{I^{2}}{2 \bar{\mu} B^{2} C^{2}}, \\
\frac{\ddot{A}}{A}+\frac{\ddot{C}}{C}+\frac{\dot{A} \dot{C}}{A C}-\frac{m^{2}}{A^{2}}=-\frac{I^{2}}{2 \bar{\mu} B^{2} C^{2}}+\xi \theta, \\
\frac{\ddot{A}}{A}+\frac{\ddot{B}}{B}+\frac{\dot{A} \dot{B}}{A B}-\frac{m^{2}}{A^{2}}=-\frac{I^{2}}{2 \bar{\mu} B^{2} C^{2}}+\xi \theta, \\
\frac{\ddot{B}}{B}+\frac{\ddot{C}}{C}+\frac{\dot{B} \dot{C}}{B C}+\frac{m^{2}}{A^{2}}=\lambda+\frac{I^{2}}{2 \bar{\mu} B^{2} C^{2}}+\xi \theta, \\
m\left(\frac{\dot{C}}{C}-\frac{\dot{B}}{B}\right)=0
\end{gathered}
$$

Equation (20) immediately gives

$$
C=n B,
$$

where $n$ is a positive integration constant. Summation of field equations (17), (18), (19) and three times (16) gives

$$
\frac{\ddot{\tau}}{\tau}=\frac{1}{2}\left[(3 \rho+\lambda)+\frac{A^{2} I^{2}}{\bar{\mu} \tau^{2}}\right]+\frac{3}{2} \xi \theta+\frac{2 m^{2}}{A^{2}} .
$$

The energy conservation equation $T_{i ; j}^{j}=0$ takes the form

$$
\dot{\rho}+\frac{\dot{\tau}}{\tau} \rho-\frac{\dot{A}}{A} \lambda=\xi \theta^{2} .
$$

Now, we consider the following equation of state for a cloud of string models ${ }^{6}$ to solve Eqs. (22) and (23).

$$
\rho=\alpha \lambda
$$

where the constant $\alpha$ is defined by

$$
\begin{aligned}
\alpha & =1 \quad(\text { geometric or Nambu string }) \\
& =(1+\omega) \quad(p-\text { string or Takabayasi string }) \\
& =-1 \quad(\text { Reddy string })
\end{aligned}
$$

where $\omega$ is a constant such that $\omega>0$.

Letelier ${ }^{4}$ pointed out that the universe could be represented by a collection of objects (galaxies), so a string dust cosmology $(\rho=\lambda)$ gives a model to investigate properties related with this fact. Zeldovich ${ }^{2}$ also explained that the string dust model could be used as a source of the gravitational field. In geometrical string model, the strings dominate and the tension along the string is equal to its energy density per unit length as we call it string dust model. Since the presence of strings in the early universe can be explained using grand unified theories, there must be different kinds of vacuum structures depending on the structure and topology of the gauge group. Letelier ${ }^{6}$ studied a model of a cloud formed by massive strings instead of geometrical strings. Each massive string is formed by a geometrical strings with particles attached along its extension. Hence, the strings that form the cloud are the generalization of Takabayasi's realistic model of strings (p-strings). Therefore, p-string is the direct consequence of Takabayasi's string model. In principle we can eliminate the strings and end up with a cloud of particles. This is a desirable property of a model of a string cloud to be used in cosmology. The above equation of state are restricted by the energy conditions ${ }^{61}$.

If we set $R=$ constant in Eq.(5), then in view of (3), we get

$$
A=d_{0} B \tau^{R / 3},
$$


where $d_{0}$ is a constant. From (2), (21) and (26), we find the following expression for metric functions in terms of $\tau$.

$$
\begin{gathered}
A=\left(d_{0}^{2} n^{-1}\right)^{1 / 3} \tau^{(3+2 R) / 9}, \\
B=\left(d_{0} n\right)^{-1 / 3} \tau^{(3-R) / 9}, \\
C=\left(d_{0}^{-1} n^{2}\right)^{1 / 3} \tau^{(3-R) / 9} .
\end{gathered}
$$

Using (24), Eq. (22) can be rewritten as

$$
\frac{\ddot{\tau}}{\tau}=\frac{1}{2}\left[\left(\frac{3 \alpha+1}{\alpha}\right) \rho+\frac{A^{2} I^{2}}{\bar{\mu} \tau^{2}}\right]+\frac{3}{2} \xi \theta+\frac{2 m^{2}}{A^{2}} .
$$

From (27) and (23) we get

$$
\dot{\rho}+\left(\rho-\frac{3+2 R}{9} \lambda\right) \frac{\dot{\tau}}{\tau}=\xi \theta \frac{\dot{\tau}}{\tau} .
$$

We further assume that the bulk viscosity is inversely proportional to the expansion scalar ${ }^{62}$ which means that the rate of cosmic expansion decreases as the viscosity increases ,i.e.,

$$
\xi \theta=\xi_{0}
$$

where $\xi_{0}$ is a positive constant. Using (24) and (32) into (31) and simplifying we get the energy density in terms of $\tau$ as

$$
\rho=\frac{9 \alpha}{9 \alpha-(3+2 R)}\left[\xi_{0}+\rho_{0} \tau^{-\left(\frac{9 \alpha-(3+2 R)}{9 \alpha}\right)}\right]
$$

where $\rho_{0}$ is a constant of integration. The string tension density is given by

$$
\lambda=\frac{9}{9 \alpha-(3+2 R)}\left[\xi_{0}+\rho_{0} \tau^{-\left(\frac{9 \alpha-(3+2 R)}{9 \alpha}\right)}\right]
$$

which may be positive for $\alpha>(3+2 R) / 9$, or negative as $0<\alpha<(3+2 R) / 9$. The rest energy density of particles, $\rho_{p}$ is given by

$$
\rho_{p}=\rho-\lambda=\frac{9(\alpha-1)}{9 \alpha-(3+2 R)}\left[\xi_{0}+\rho_{0} \tau^{-\left(\frac{9 \alpha-(3+2 R)}{9 \alpha}\right)}\right],
$$

For $\rho$ and $\rho_{p}$ to be positive we must have $\alpha>\frac{3+2 R}{9}$. It is to be noted that $\frac{3+2 R}{9}$ is always positive, therefore, the equation of state parameter $\alpha$ must be considered as a positive. Hence, we find that the Reddy string model $(\alpha=-1)$ is not possible in this case. Specially, for the choice of $R=0$, where the model isotropizes at late time, we find $\alpha>1 / 3$. Therefore, this model is suitable to describe the geometrical and p-string models. The energy density, string tension density and particle energy density are decreasing functions of $\tau$ and tend to a constant as $\tau \rightarrow \infty$. It is observed that $\rho_{p}$ vanishes for geometrical string model $(\alpha=1)$ and therefore it behaves as a cloud of geometric string $(\rho=\lambda)$.

Using (27), (32) and (33), Eq. (30) now read as

$$
\ddot{\tau}=\frac{9}{2} \frac{(3 \alpha+1) \rho_{0}}{[9 \alpha-(3+2 R)]} \tau^{\frac{3+2 R}{9 \alpha}}+\frac{\left(d_{0}^{2} n^{-1}\right)^{2 / 3} I^{2}}{2 \mu} \tau^{\frac{4 R-3}{9}}+\frac{3}{2}\left(\frac{18 \alpha-2 R}{9 \alpha-3-2 R}\right) \xi_{0} \tau+\frac{2 m^{2}}{\left(d_{0}^{2} n^{-1}\right)^{2 / 3}} \tau^{\frac{3-4 R}{9}},
$$

This equation allows the following first integral

$$
\dot{\tau}=\sqrt{C_{1} \tau^{\frac{3+2 R+9 \alpha}{9 \alpha}}+C_{2} \tau^{\frac{4 R+6}{9}}+C_{3} \tau^{2}+C_{4} \tau^{\frac{12-4 R}{9}}+C_{5}}
$$

where $C_{1}=\left(\frac{81 \alpha(3 \alpha+1) \rho_{0}}{[9 \alpha-(3+2 R)][9 \alpha+(3+2 R)]}\right), \quad C_{2}=\frac{9\left(d_{0}^{2} n^{-1}\right)^{2 / 3} k}{(4 R+6)}, \quad k=\frac{I^{2}}{\mu}, \quad C_{3}=\frac{3}{2} \frac{(18 \alpha-2 R) \xi_{0}}{[9 \alpha-(3+2 R)]}, \quad C_{4}=\frac{36 m^{2}}{\left(d_{0}^{2} n^{-1}\right)^{2 / 3}(12-4 R)}$, and $C_{5}=$ constant.

We observe that Eq.(36) is a function of $\tau$ only, i.e.,

$$
\ddot{\tau}=F(\tau) .
$$

From the mechanical point of view, Eq. (38) can be interpreted as equation of motion of a single particle with unit mass under the force $F(\tau)$. Then, the following first integral exists

$$
\dot{\tau}=\sqrt{2[\epsilon-u(\tau)]}
$$

where $\epsilon$ can be viewed as energy level and $u(\tau)=-\int F(\tau) d \tau$ is the potential of the force $F$. A comprehensive description concerning potential may be found in ref. ${ }^{63}$. Comparing (37) and (39), we find $\epsilon=C_{5} / 2$, and hence the potential is given by

$$
u(\tau)=-\frac{1}{2}\left(C_{1} \tau^{\frac{3+2 R+9 \alpha}{9 \alpha}}+C_{2} \tau^{\frac{4 R+6}{9}}+C_{3} \tau^{2}+C_{4} \tau^{\frac{12-4 R}{9}}\right) .
$$


Equation (37) admits the following solution in quadrature form as

$$
\int \frac{d \tau}{\sqrt{C_{1} \tau^{\frac{3+2 R+9 \alpha}{9 \alpha}}+C_{2} \tau^{\frac{4 R+6}{9}}+C_{3} \tau^{2}+C_{4} \tau^{\frac{12-4 R}{9}}+C_{5}}}=t+t_{0},
$$

where $t_{0}$ is a constant of integration and we take it as a zero for simplicity.

\section{Solution for String Models}

We observe that it is too difficult to solve (41), in general. Therefore, we limit ourselves to investigate the behavior of the solutions for large $t$, i.e., to investigate the possibility of the isotropic solutions where all the scale factors have a similar behavior. For this we consider $R=0$ in the above solutions which we discuss for the various string models in the following subsections.

\subsection{Geometric string model $(\alpha=1)$}

Let us find the solution for viscous fluid with and without magnetic field in the following subsections.

\subsubsection{Viscous fluid solution with magnetic field}

For $R=0$ and $\alpha=1$, Eq. (41) reduces to

$$
\int \frac{d \tau}{\sqrt{\left(C_{1}+C_{4}\right) \tau^{\frac{4}{3}}+C_{2} \tau^{\frac{2}{3}}+C_{3} \tau^{2}}}=t .
$$

For $\left(C_{1}+C_{4}\right)^{2}<4 C_{2} C_{3}$, integrating (42), we get

$$
\tau=\left[\frac{1}{2 C_{3}}\left(\sqrt{4 C_{2} C_{3}-\left(C_{1}+C_{4}\right)^{2}} \sinh \left(\frac{2}{3} \sqrt{C_{3}} t\right)-\left(C_{1}+C_{4}\right)\right)\right]^{\frac{3}{2}}, \quad C_{3} \neq 0
$$

For small $t$, we have $\sinh \left(\frac{2}{3} \sqrt{C_{3}} t\right) \approx \frac{2}{3} \sqrt{C_{3}} t$. Therefore, Eq. (43) gives

$$
\tau=\left(k_{1} t-k_{2}\right)^{3 / 2}
$$

where $k_{1}=\sqrt{\frac{4 C_{2} C_{3}-\left(C_{1}+C_{4}\right)^{2}}{9 C_{3}}}$ and $k_{2}=\frac{\left(C_{1}+C_{4}\right)}{2 C_{3}}$. We observe that $\tau$ becomes imaginary at $t=0$. Thus, for reality of the model, we must have $t \geq \frac{k_{2}}{k_{1}}$.

The metric coefficients for isotropic regime have the form

$$
A \sim B \sim C \sim\left(k_{1} t-k_{2}\right)^{\frac{1}{2}},
$$

and consequently the Hubble parameter is given by

$$
H=\frac{k_{1}}{2\left(k_{1} t-k_{2}\right)} .
$$

For this isotropic regime the density of strings is given by

$$
\rho=\frac{3}{2}\left[\xi_{0}+\frac{\rho_{0}}{k_{1} t-k_{2}}\right]=\lambda .
$$

We observe that $\rho$ and $\lambda$ remains positive throughout the evolution and become infinite at the initial epoch at $t=\frac{k_{2}}{k_{1}}$. However, $\rho$ and $\lambda$ decrease with time for $t>\frac{k_{2}}{k_{1}}$ and approach to a constant value, $\frac{3}{2} \xi_{0}$ as $t \rightarrow \infty$. We observe that this constant value of $\rho$ is due to the viscous term, $\xi_{0}$. It means that the bulk viscosity prevents the universe to be empty at late times of its evolution.

The potential (40) in terms of $t$ takes the form

$$
u(t)=-\frac{1}{2}\left[C_{3}\left(k_{1} t-k_{2}\right)^{3}+\left(C_{1}+C_{4}\right)\left(k_{1} t-k_{2}\right)^{2}+C_{3}\left(k_{1} t-k_{2}\right)\right] .
$$

The behavior of potential for viscous fluid (bold line) is shown in figure 1 . 


\subsubsection{Viscous fluid solution without magnetic field}

For $\alpha=1$ and in the absence of magnetic field $(k=0)$, Eq.(41) becomes

$$
\int \frac{d \tau}{\sqrt{\left(C_{1}+C_{4}\right) \tau^{\frac{4}{3}}+C_{3} \tau^{2}}}=t
$$

which on integration it gives

$$
\tau=\left(\frac{C_{1}+C_{4}}{2 C_{3}}\right)\left[\cosh \left(\frac{2 \sqrt{C_{3}}}{3} t\right)-1\right]^{\frac{3}{2}}, \quad C_{3} \neq 0
$$

For small $t$, Eq.(50) gives

$$
\tau=\left[\frac{2\left(C_{1}+C_{4}\right)}{9}\right]^{\frac{3}{2}} t^{3}
$$

The metric coefficients for isotropic regime have the form

$$
A \sim B \sim C \sim t
$$

and consequently the Hubble parameter is given by

$$
H=\frac{1}{t}
$$

For this isotropic regime the density of strings is given by

$$
\rho=\frac{3}{2}\left[\xi_{0}+\frac{9 \rho_{0}}{2\left(C_{1}+C_{4}\right)} \frac{1}{t^{2}}\right]=\lambda .
$$

We find that the energy density remains positive through out the evolution of the universe. It is infinite at initial epoch $t=0$ and decreases with time, and ultimately attains a constant value, $\frac{3 \xi_{0}}{2}$ in late time expansion of the universe. Therefore, the bulk viscosity prevents the universe to be empty during late time of evolution. We also find that the energy density attains the same constant value with and without magnetic field in late time of evolution which shows that the magnetic field has no effect in late time epoch.

The potential (40) in terms of $t$ takes the form

$$
u(t)=-\frac{2\left(C_{1}+C_{4}\right)^{3}}{81}\left(1+\frac{2 C_{3}}{9} t^{2}\right) t^{4} .
$$

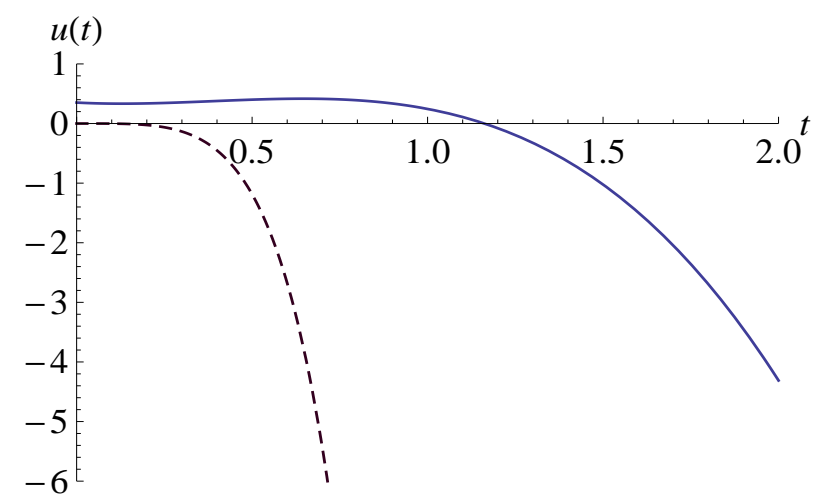

Figure 1. Potential versus time for viscous fluid with (bold line) and without (dashed line) magnetic field

Figure 1 plots the potential with respect to time in the presence of viscous fluid and magnetic field (bold line) and with viscous fluid only (dashed line). We have used the numerical value of various constants such as $\rho_{0}=1$, $d_{0}=1, n=1, m=1, \xi_{0}=1, I=2$ or 0 and $\bar{\mu}=1.00001$. We observe that $\mu(t)$ starts from origin and goes to negative through out the evolution in the presence of viscous without magnetic field where as it starts from some positive value and remains positive for finite time of interval and then becomes negative nature with respect to time $t$ in magnetized viscous fluid. Therefore, we can say that viscous and magnetic field have the effects on the early evolution of the universe. However, the effects become negligible during late time evolution. The string model with viscous fluid may be described as an acceptable model. 


\subsection{Takabayashi string model $(\alpha=1+\omega)$}

In this case, Eq.(41) reduces to

$$
\int \frac{d \tau}{\sqrt{C_{1} \tau^{\frac{3 \omega+4}{3 \omega+3}}+C_{2} \tau^{\frac{2}{3}}+C_{3} \tau^{2}+C_{4} \tau^{\frac{4}{3}}}}=t
$$

One can observe that it is very difficult to find a general solution of $\tau$ in terms of $t$. Therefore, we express $\rho, \lambda$ and $\rho_{p}$ in terms of $\tau$ as

$$
\begin{gathered}
\rho=\frac{3(1+\omega)}{3 \omega+2}\left[\xi_{0}+\rho_{0} \tau^{-\frac{2+3 \omega}{3(1+\omega)}}\right], \\
\lambda=\frac{3}{3 \omega+2}\left[\xi_{0}+\rho_{0} \tau^{-\frac{2+3 \omega}{3(1+\omega)}}\right], \\
\rho_{p}=\frac{3 \omega}{3 \omega+2}\left[\xi_{0}+\rho_{0} \tau^{-\frac{2+3 \omega}{3(1+\omega)}}\right],
\end{gathered}
$$

As $\omega>0$, we find that $\rho, \lambda$ and $\rho_{p}$ remain positive through out the evolution of the universe. These physical parameters are decreasing function of $\tau$ and become constant for large $t$ due to the bulk viscosity. The geometrical string model may be recovered for $\omega=0$ as discussed in Sect. 3.1. The potential in this is given by

$$
u(\tau)=-\frac{1}{2}\left[C_{1} \tau^{\frac{4+3 \omega}{3(1+\omega)}}+C_{2} \tau^{\frac{2}{3}}+C_{3} \tau^{2}+C_{4} \tau^{\frac{4}{3}}\right] .
$$

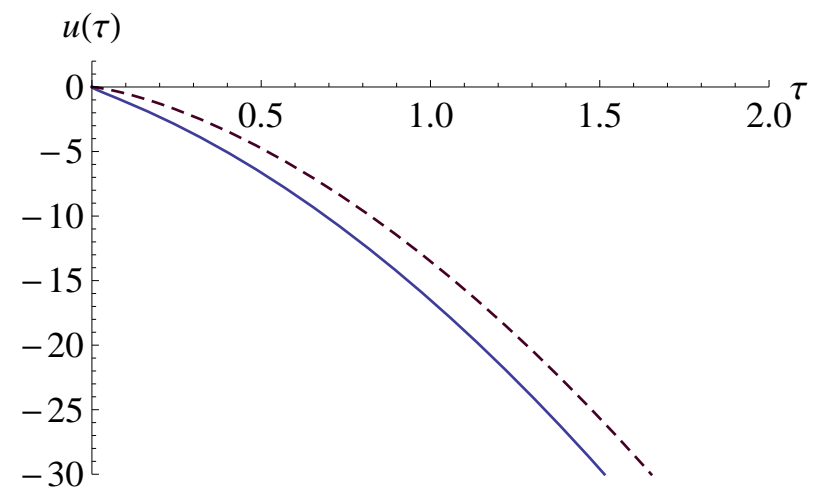

Figure 2. Potential versus time for viscous fluid solution with (bold lines) and without (dashed lines) magnetic field.

Figure 2 illustrates the behavior of potential $\mu(t)$ with respect to $\tau$ for $\rho_{0}=1, d_{0}=1, n=1, m=1, \xi_{0}=1, I=2$, or $0, \bar{\mu}=1.00001$ and $\omega=1$. It is clear that potential remains negative and decreases rapidly throughout the evolution of the universe for viscous fluid with and without magnetic field. It decreases rapidly due to the viscous fluid only.

\section{Conclusion}

We have studied anisotropic Bianchi $\mathrm{VI}_{0}$ string cosmological model with viscous fluid and magnetic field in general relativity by taking certain physical assumptions. Since viscous fluid and magnetic field have cosmological origin, it is interesting to discuss the viscous and magnetic field effects on the expansion history of the universe in early and late stages of evolution in string cosmology. The Einstein's field equations have been solved exactly for geometrical string model for viscous fluid with and without magnetic field whereas a general quadrature form of average scale factor has been found in Takabayashi string model. The solutions present interesting features in the presence of magnetized viscous fluid and in the presence of viscous fluid only. We have found that the viscous term affects the solutions much rapid as compare to magnetic field. We have also analyzed the behavior of various physical parameters graphically in the presence of magnetized viscous fluid and bulk viscous fluid without magnetic field.

In geometrical string model we have observed that proper energy density remains positive through out the evolution and attains the same constant value during late time for viscous fluid with and without magnetic field. Hence, the presence of viscous term prevents to be empty in its future evolution. The classical potential changes its behavior rapidly due to the bulk viscous term. It is negative through out the evolution in the absence of magnetic field but it is positive for some finite time and after that it shows the negative nature during late time.

In Takabayashi model we have found a quadrature form of average volume which is too difficult to solve, in general. The solution for the proper energy density, string tension density and particle energy density in terms of 
average scale factor have been represented. These physical parameters approach to a constant value asymptotically as $t \rightarrow \infty$ due to the bulk viscosity.

We have also discussed the potential with respect to time in each string model and have observed that the classical potential changes its behavior rapidly due to the bulk viscous term. In geometrical string model it remains negative and decreases rapidly in viscous fluid solution without magnetic field. In Takabayashi string model it remains negative in both cases but the effect of viscous fluid with and without magnetic field vary rapidly. Thus, we conclude that the bulk viscous fluid with and without magnetic field plays an important role in the evolution of the universe in anisotropic models as analyzed in this paper.

\section{REFERENCES}

[1] T.W.B. Kibble, J. Phys. A 9, 1387 (1976)

[2] Y.B. Zel'dovich, Mon. Not. R. Astron. Soc. 192, 663 (1980).

[3] A. Vilenkin, Phys. Rev. D 24, 2082 (1981).

[4] P.S. Letelier, Phys. Rev. D 20, 1294 (1979)

[5] J. Stachel, Phys. Rev. D 21, 2171 (1980)

[6] P.S. Letelier, Phys. Rev. D 28, 2414 (1983)

[7] D.R. Matraverse, Gen. Relativ. Grav. 20, 279 (1988)

[8] C.W. Misner, Nature 214, 40 (1967)

[9] C.W. Misner, Astrophys. J. 151, 431 (1968)

[10] R.A. Matzner, Astrophys. J. 157, 1085 (1969)

[11] A.G. Doroshkevich, Y.B. Zeldovich, I.D. Nivokov, Zh. Eksp. Theor. Fiz. 53, 644 (1967)

[12] J.M. Stewart, Mon. Not. Roy. Soc. 145, 347 (1969)

[13] C.B. Collins, J.M. Stewart, Mon. Not. Roy. Soc. 153, 419 (1971)

[14] M.A. Melvin, Ann. N.Y. Acad. Sci. 262, 2531975.

[15] E. Asseo, H. Sol, Phys. Rep. 6, 148 (1987)

[16] M.S. Madsen, MNRAS 237, 109 (1989)

[17] A.M. Wolfe, K. Lanzetta, A.L. Oren, Astrophys. J. 388, 17 (1992)

[18] R. Kulsrud, R. Cen, J.P. Ostriker, D. Ryu, Astrophys. J. 380, 481 (1997)

[19] J.D. Barrow, Phys. Rev. D 55,7451 (1997)

[20] D.R. Matravers, C.G. Tsagas, Phys. Rev. D 62, 103519 (2000) 
[21] J.D. Barrow, Mon. Not. R. Astron. Soc. 211, 221 (1984).

[22] D. Lorentz, Astrophys. Space Sci. 85, 69 (1982).

[23] S.R. Roy and J.P. Singh, Acta Physic Austriaca 5, 57 (1983).

[24] S.R. Roy, J.P. Singh, S. Narain, Astrophys. Space Sci. 111, 389 (1985).

[25] ShriRam: J. Math. Phys. 27, 650 (1986).

[26] C. Uggla and K. Rosquist, Class. Quantum Gravity 5, 767 (1988).

[27] ShriRam: Int. J. Theor. Phys. 28, 917 (1989).

[28] A.A. Coley and K. Dunn, Astrophs. J. 348, 26 (1990).

[29] S.R. Roy and S.K. Banerjee, Gen Relativ. Gravit. 24, 1117 (1992).

[30] S.R. Roy and S.K. Banerjee, Gen Relativ. Gravit. 28, 27 (1992).

[31] ShriRam and P. Singh, Astrophys. Space Sci. 200, 35 (1993).

[32] G. Mohanthy, S.K. Sahu, Astrophys. Space Sci. 288, 611 (2003).

[33] M. Weaver, Class. Quantum Gravity 17, 421 (2000).

[34] M. Sharif and M. Zubair, Int. J. Mod. Phys. D 19, 1957 (2010).

[35] M. Sharif and M. Zubair, Astrophys. Space Sci. 339, 45 (2012).

[36] K.D. Krori, T. Chaudhury, C.R. Mahanta, A. Mazumdar, Gen. Relativ. Grav. 22, 123 (1990)

[37] S. Chakraborty, Indian J. Pure Appl. Phys. 29, 31 (1991).

[38] R. Tikekar, L.K. Patel, Pramana J-Phys 42, 483 (1994).

[39] M.B. Ribeiro, A.K. Sanyal, J. Math. Phys. 28, 657 (1987).

[40] A.K. Banerjee, A.K. Sanyal, Gen. Relativ. Gravit. 18, 1251 (1986).

[41] R. Bali, D.R. Jain, Int. J. Theor. Phys. 28, 903 (1989).

[42] X.X. Wang, Astrophys. Space Sci. 293, 433 (2004).

[43] X.X. Wang, Chin. Phys. Lett. 23, 1702 (2006).

[44] M.K. Yadav, A. Pradhan, S.K. Singh, Astrophys. Space Sci. 311, 423 (2007).

[45] R. Bali, A. Pradhan, H. Amirhashchi, Int. J. Theor. Phys. 47, 2594 (2008). 
[46] R. Bali, R. Banerjee, S.K. Banerjee, Astrophys. Space Sci. 317, 21 (2008).

[47] S.K. Tripathy, S.K. Nayak, S.K. Sahu, T.R. Toutray, Astrophys. Space Sci. 323, 91 (2009).

[48] S.K. Tripathy, S.K. Nayak, S.K. Sahu, T.R. Toutray, Astrophys. Space Sci. 323, 281 (2009).

[49] S.K. Tripathy , D. Behera, Astrophys. Space Sci. 330, 191 (2010).

[50] A. Pradhan, R. Bali, Eect. J. Theor. Phys. 19, 91 (2008).

[51] M. Sharif, S. Waheed, Int. J. Mod. Phys. D 55, 21500 (2012).

[52] B. Saha, M. Visinescu, Astrophys. Space Sci. 315, 99 (2008)

[53] B. Saha, V. Rikhvitsky, M. Visinescu, Cent. Eur. J. Phys. 8, 113 (2010)

[54] V. Rikhvitsky, B. Saha, M. Visinescu, Astrophys. Sapce. Sci. 339, 371, (2012)

[55] B. Saha, M. Visinescu, Rom. J. Phys. 55, 1064 (2010)

[56] C.P. Singh, Astrophys. Space Sci. 343, 773 (2013)

[57] C.P.Singh, Int. J. Theor. Phys. DOI:10.1007/s 10773-013-1952-1, (2013).

[58] J.D. Barrow, Phys. Rev. D 55, 7451 (1997).

[59] L.D. Landau, E.M. Lifshitz, Fluid Mechanics, Pergamon Press, Oxford,p.505. (1936)

[60] A. Linchnerowicz, Relativistic Hydrodynamics and Magneto Hydrodynam ics, Benjamin, New York, p.13 (1967)

[61] A. Banerjee, A.K. Sanyal and S. Chakraborty, Pramana-J. Phys. 34, 1 (1990).

[62] B. Saha, Mod. Phys. Lett. A 20, 2127 (2005)

[63] B. Saha, T. Boyadjiev, Phys. Rev. D 69, 124010 (2004). 